\title{
Canadian Public Health Laboratory Network position statement: Nonculture based diagnostics for gastroenteritis and implications for public health investigations
}

\author{
B Berenger ${ }^{1,2}$, L Chui ${ }^{1}$, AR Reimer ${ }^{3^{*}}$, V Allen 4 , D Alexander ${ }^{5}$, M-C Domingo ${ }^{6}$, D Haldane ${ }^{7}$, L Hoang ${ }^{8}$, \\ P Levett ${ }^{9}$, A MacKeen ${ }^{10}$, D Marcino ${ }^{10}$, C Sheitoyan-Pesant ${ }^{11}$, G Zahariadis ${ }^{12}$ on behalf of Canadian \\ Public Health Laboratory Network
}

\begin{abstract}
As clinical laboratories transition to using culture-independent detection test (CIDT) panels for cases of acute gastroenteritis, culture of clinical specimens is becoming less common. The reduction in bacterial cultures available for public health activities is expected to hinder surveillance and outbreak response by public health laboratories at the local, provincial, national and international levels. These recommendations are intended to serve as guidelines for the implementation of CIDT panels in frontline laboratories in Canada. The United States of America has already seen a significant reduction in culture of stool specimens despite the Association of Public Health Laboratories recommendation to perform reflex culture on positive CIDT specimens. Priority public health organisms addressed in these Canadian guidelines include Shiga toxin-producing Escherichia coli, Shigella and Salmonella and, under regional circumstances, other organisms such as Campylobacter jejuni/coli and Yersinia enterocolitica. These recommendations suggest active engagement between primary diagnostic laboratories and provincial public health laboratories to determine the workflow and protocols for reflex or parallel culture. Consequently, notifiable disease definitions will also need modification, with consultation of all stakeholders. Stakeholders need to work together to enhance recovery of bacterial isolates with best practices used for stool transport and storage.
\end{abstract}

\section{Affiliations}

${ }^{1}$ Alberta Provincial Laboratory for Public Health, Calgary, AB

${ }^{2}$ Calgary Laboratory Services, Calgary, $A B$

${ }^{3}$ National Microbiology Laboratory, Winnipeg, MB

${ }^{4}$ Public Health Ontario, Toronto, $\mathrm{ON}$

${ }^{5}$ Cadham Provincial Laboratory, Winnipeg, MB;

${ }^{6}$ Laboratoire de Santé Publique du Québec, Sainte-Anne-de-Bellevue, QC

${ }^{7}$ Queen Elizabeth II Health Science Centre, Halifax, NS

${ }^{8}$ BC Centre for Disease Control, Vancouver, BC

9 Saskatchewan Disease Control Laboratory, Regina, SK

${ }^{10}$ Canadian Public Health Laboratory Network, Winnipeg, MB

${ }^{11}$ Centre Hospitalier Universitaire Dr Georges-L.-Dumont, Moncton, NB

12 Provincial Public Health Laboratory, Eastern Health Microbiology Services, St. John's, $\mathrm{NL}$

*Correspondence: aleisha. reimer@canada.ca

\section{Introduction}

Diagnostic testing of enteric bacteria informs both individual clinical decisions and serves as a critical surveillance mechanism to detect outbreaks, protect populations and to mitigate further spread of disease. Generally, front line diagnostic microbiology laboratories detect the presence of enteric pathogens for clinical purposes and forward isolates to a provincial public health laboratory for confirmation and typing. Typing results are then compared with databases of other isolates via PulseNet Canada (and internationally, as needed) to inform public health actions and interventions within and across jurisdictions. Results are also integrated with sentinel-site surveillance programs targeting broad food safetyrelated issues (i.e., FoodNet Canada). Clusters of clinical cases and outbreaks detected and linked by genetic comparison of isolates from patients, the environment, food and animals drive most foodborne disease outbreak investigations. From 2008 to 2014, 115 foodborne outbreaks were detected in Canada via these surveillance mechanisms (1). Submission of isolates to a provincial public health laboratory also enables access to information on predominant circulating strains and antibiotic resistant profiles.

This crucial public health surveillance and protection mechanism and its effectiveness is under threat from the implementation of culture-independent diagnostic tests (CIDTs), which were 
developed for diagnostic application in the front line laboratory setting and which bypass the need to recover the bacteria necessary for public health surveillance (2). In the United States, many laboratories have discontinued culture altogether and the Centers for Disease Control and Prevention has reported a drastic reduction in the submission of isolates to state public health laboratories since the introduction of CIDT (3). The United States FoodNet surveillance system found that there was no reflex culture attempted in $35.6 \%$ of bacterial gastroenteritis cases diagnosed by CIDT (4) and that CIDT-positive diagnoses that were not confirmed by culture increased by $114 \%$ in 2016 compared to the previous three years (5).

In response to this decrease in culture results, the Association of Public Health Laboratories has released interim recommendations for CIDT testing. In brief, these interim recommendations for enteric pathogens ask each clinical laboratory to:

- Continue to obtain and submit isolates of foodborne pathogens to local and state public health laboratories

- Submit the CIDT-positive specimen to a public health laboratory if unable to culture the isolates themselves

- Maintain effective and open communication with public health laboratories in their state or jurisdiction, including notifying the public health laboratories of the intent to implement a CIDT for foodborne pathogens, and to delineate the increased responsibilities of state public health departments and laboratories as well as national authorities

This guideline presents the recommendations of the Canadian Public Health Laboratory Network for the implementation of CIDT in Canadian laboratories.

\section{Recommendations}

\section{Primary recommendation}

1. If a CIDT is used as a primary screening tool for bacterial gastroenteritis, culture is to be performed on stools positive for a bacterial pathogen.

Culture, using appropriate methods, should be performed on CIDT-positive stools for bacterial pathogens of public health significance and when an isolate is required for antibiotic susceptibility testing to guide clinical treatment.

In Canada, organisms of public health significance include Shiga toxin-producing Escherichia coli, Shigella and Salmonella. These organisms are currently part of surveillance performed at provincial public health laboratories and the Public Health Agency of Canada's National Microbiology Laboratory. Genotyping (i.e., using pulsed-field gel electrophoresis and whole-genome sequencing) on pure cultures of these species is required for cluster and outbreak detection through PulseNet. Depending on the jurisdiction that the laboratory services, additional organisms may need to be submitted to the public health laboratory (e.g., Campylobacter jejuni/coli and Yersinia enterocolitica).

\section{Additional recommendations}

2. Consultation between primary diagnostic laboratories and provincial public health laboratories should occur in order to define the roles and responsibilities that optimize surveillance workflow.

Targeted reflex culture on CIDT-postive stools, or parallel culture should be performed by the designated laboratory. Specific protocols should be developed at the regional level.

3. To determine if notifiable disease definitions need to be modified following the implementation of CIDT, consultation between stakeholders (public health authorities, public health laboratories, primary diagnostic laboratories and clinicians) should occur.

Depending on the province, notifiable disease case definitions may require culture of a bacterial agent to identify a case and trigger investigation; therefore, modification of case definitions may need to occur to account for cases identified with CIDT. Focus should be placed on determining how to deal with cases that are CIDT-positive and culture-negative. At the time of writing, the evidence is insufficient to determine if molecular detection with culture negativity indicates infection. A CIDT-positive and culture-negative result may indicate sampling error, low organism burden or dead organism due to antibiotic administration or loss of viability (e.g., due to long transportation time from collection to culture).

We recommend that CIDT-positive and culture-negative cases are deemed probable cases under provincial and national notifiable disease definitions. In cases where it is necessary to determine if the patient has viable organisms or confirms that this is indeed a case, repeat culture of the initial stool specimen is recommended. A second stool specimen for repeat culture and CIDT testing may also be useful in trying to obtain an isolate for typing. If resources are available, a confirmatory polymerase chain reaction (PCR) with a different target should be used if a false-positive or unusual molecular result is suspected.

4. Consult with stakeholders to determine the appropriate frontline CIDT panel and which front line cultures need to be maintained.

Many CIDT commercial panels do not test for some of the organisms that are required for routine stool culture reports and different CIDT panels may test for different organisms. Thus, the specific panel for the specific population serviced by the relevant laboratories must be determined. Primary culture screening for some organisms may still be needed. The indications and locations of primary culture for these additional organisms need to be determined in advance of CIDT implementation.

Some pathogens screened for by culture may be of questionable pathogenicity (e.g., Aeromonas spp.); laboratories and stakeholders may or may not choose to maintain testing for these organisms. Alternatively, cultures of these organisms could be required under prescribed circumstances.

The CIDT test panels may also include other pathogens not included in a routine bacterial gastroenteritis culture, such as viruses or Clostridium difficile. The appropriateness of testing and reporting of multiple pathogens in different clinical scenarios needs to be carefully considered. It should be noted 
that asymptomatic patients can be positive for some of these pathogens and the clinical impact on the patient needs to be considered (6).

5. To enhance recovery of bacterial isolates, best practices should be used for stool transport and storage.

A variety of pre-analytical factors can impair recovery of bacterial isolates, including long transportation times and storage at ambient temperatures. Cary-Blair, modified Cary-Blair and similar transport media are often recommended to improve recovery of organisms and should be considered when samples are collected at remote sites or turnaround times are long. Storage at $4^{\circ} \mathrm{C}$ will prevent overgrowth and enhance recovery of organisms. For long-term storage, freezing is preferable to $4^{\circ} \mathrm{C}$. If practical, labs may consider storing stool specimens in the freezer while awaiting CIDT results $(7,8)$.

\section{Conclusion}

These guidelines are the critical first step to moderating the public health impact of acute gastroenteritis assays on the market in Canada. It is imperative that each front line and public health laboratory engage with their counterpart laboratories to develop a test strategy that serves both clinical diagnostics and public health purposes.

\section{Authors' statement}

All authors were members of the Canadian Public Health Laboratory Network (CPHLN) Culture Independent Diagnostic Testing Working Group. This group was chaired by AR Reimer (Federal) and V Allen (Provincial).

\section{Conflict of Interest}

None.

\section{Acknowledgements}

The authors would like to acknowledge members of the Canadian Public Health Laboratory Network Executive and Secretariat for their advice and guidance in the development of this position statement.

\section{Funding}

Funding for the Canadian Public Health Laboratory Network Secretariat is provided by the National Microbiology Laboratory.

\section{References}

1. Bélanger $P$, Tanguay F, Hamel M, Phypers M. An overview of foodborne outbreaks in Canada reported through Outbreak Summaries: 2008-2014. Can Commun Dis Rep 2015;41(11):254-262. https://www.canada.ca/en/publichealth/services/reports-publications/canada-communicabledisease-report-ccdr/monthly-issue/2015-41/ccdr-volume-4111-november-5-2015-foodborne-illness/ccdr-volume-41-11november-5-2015-foodborne-illness.html

2. Shea S, Kubota KA, Maguire H, Gladbach S, Woron A, Atkinson-Dunn $\mathrm{R}$ et al. Clinical Microbiology Laboratories' Adoption of Culture-Independent Diagnostic Tests Is a Threat to Foodborne-Disease Surveillance in the United States. J Clin Microbiol 2016 Dec;55(1):10-9. DOI (http://dx.doi. org/10.1128/JCM.01624-16). PubMed (https://www.ncbi.nlm. nih.gov/entrez/query.fcgi?cmd=Retrieve\&db=PubMed\&lis t_uids=27795338\&dopt=Abstract).

3. Iwamoto M, Huang JY, Cronquist AB, Medus C, Hurd S, Zansky $S$ et al.; Centers for Disease Control and Prevention (CDC). Bacterial enteric infections detected by culture-independent diagnostic tests--FoodNet, United States, 2012-2014. MMWR Morb Mortal Wkly Rep 2015 Mar;64(9):252-7. PubMed (https://www.ncbi.nlm.nih.gov/entrez/query.fcgi?cmd=Retrieve $\& d b=$ PubMed\&list_uids=25763878\&dopt=Abstract).

4. Huang JY, Henao OL, Griffin PM, Vugia DJ, Cronquist AB, Hurd $\mathrm{S}$ et al. Infection with Pathogens Transmitted Commonly Through Food and the Effect of Increasing Use of CultureIndependent Diagnostic Tests on Surveillance--Foodborne Diseases Active Surveillance Network, 10 U.S. Sites, 20122015. MMWR Morb Mortal Wkly Rep 2016 Apr;65(14):368-71. DOI (http://dx.doi.org/10.15585/mmwr.mm6514a2). PubMed (https://www.ncbi.nlm.nih.gov/entrez/query.fcgi?cmd=Retrieve $\& d b=$ PubMed\&list_uids=27077946\&dopt=Abstract).

5. Marder EP, Cieslak PR, Cronquist AB, Dunn J, Lathrop S, Rabatsky-Ehr T et al. Incidence and Trends of Infections with Pathogens Transmitted Commonly Through Food and the Effect of Increasing Use of Culture-Independent Diagnostic Tests on Surveillance - Foodborne Diseases Active Surveillance Network, 10 U.S. Sites, 2013-2016. MMWR Morb Mortal Wkly Rep 2017 Apr;66(15):397-403. DOI (http://dx.doi. org/10.15585/mmwr.mm6615a1). PubMed (https://www.ncbi. $\mathrm{nlm}$.nih.gov/entrez/query.fcgi?cmd=Retrieve\&db=PubMed\&li st_uids $=28426643 \&$ dopt $=$ Abstract .

6. Alasmari F, Seiler SM, Hink T, Burnham CA, Dubberke ER. Prevalence and risk factors for asymptomatic Clostridium difficile carriage. Clin Infect Dis 2014 Jul;59(2):216-22. DOI (http://dx.doi.org/10.1093/cid/ciu258). PubMed (https://www. ncbi.nlm.nih.gov/entrez/query.fcgi?cmd=Retrieve\&db=PubMe d\&list_uids=24755858\&dopt=Abstract).

7. Wells JG, Morris GK. Evaluation of transport methods for isolating Shigella spp. J Clin Microbiol 1981 Apr;13(4):789-90. PubMed (https://www.ncbi.nlm.nih.gov/entrez/query.fcgi?cmd $=$ Retrieve \&db=PubMed\&list_uids=6785312\&dopt=Abstract).

8. Wang WL, Reller LB, Smallwood B, Luechtefeld NW, Blaser MJ. Evaluation of transport media for Campylobacter jejuni in human fecal specimens. J Clin Microbiol 1983 Oct;18(4):803-7. PubMed (https://www.ncbi.nlm.nih.gov/entrez/query.fcgi?cmd $=$ Retrieve $\& d b=$ PubMed\&list_uids=6355160\&dopt=Abstract). 